\title{
Thermodynamics of the Limiting Cases of the XXZ Model Without Bethe Ansatz
}

\author{
Onofre Rojas ${ }^{1}$, S.M. de Souza ${ }^{2}$, E.V. Corrêa Silva ${ }^{3}$, and M.T. Thomaz ${ }^{1}$ \\ ${ }^{1}$ Instituto de Física, Universidade Federal Fluminense \\ Av. Gal. Milton Tavares de Souza s/n., CEP 24210-340, Niterói, RJ, Brazil \\ ${ }^{2}$ Departamento de Ciências Exatas, Universidade Federal de Lavras \\ Caixa Postal 37, CEP 37200-000, Lavras, MG, Brazil \\ ${ }^{3}$ Instituto de Física, Universidade do Estado do Rio de Janeiro \\ R. São Francisco Xavier no. 524, 3? andar, CEP 20550-013, Rio de Janeiro, RJ, Brazil
}

Received on 18 June, 2001.

\begin{abstract}
The Heisenberg XXZ model is a chain model with nearest-neighbor interactions. Its thermodynamics is exactly obtained via Bethe ansatz. Recently, we developed a method to derive the hightemperature expansion of the grand potential per site of translationally invariant chain models, with periodic boundary conditions. Here we apply this approach to the XXZ model with periodic boundary conditions for the Ising limit case $(t=0)$ and the free fermion case $(\Delta=0$ and $h=0)$, obtaining results in agreement with the literature. In this new way of obtaining the coefficients of the high-temperature expansion of the grand potential, the coefficients are derived from an auxiliary function written only in terms of open connected sub-chains.
\end{abstract}

\section{Introduction}

In many aspects exactly solvable models are very important in physics. They can, for example, give us insights about more realistic models. The exactly solvable models and their limits have also been applied along decades as tests of applicability of new approaches.

Chain models as the Heisenberg XXZ model [1] and the one-dimensional Hubbard model[2, 3, 4, 5] are exactly solvable models due to their integrability property. The Bethe ansatz has been applied to writing the thermodynamic functions of those models as non-linear integral equations (NLIE) (see the references mentioned above). The numerical analysis of those NLIE has been under study since the seventies. Those numerical analyses are very involved and in 1995 Destri and de Vega[6] got a high temperature expansion from the NLIE of the free energy of the XXZ model.

The XXZ model has two limiting cases: $i$ ) the Ising model [7] and $i i)$ the free fermion model [8], whose respective free energies have closed analytical expressions.

Recently, we proposed a new approach to obtaining the high temperature expansion of the free energy in the thermodynamic limit of chain models[9], the results of which are valid for both fermionic and bosonic models, and which does not rely on the Bethe ansatz (a keystone to all previous approaches to integrable models). In the revised version of reference [9], we applied this method to get the $\beta$-expansion of the free energy of the XXZ model. We showed that the $\beta^{3}$ term of the expression derived by Destri and de Vega[6] had to be fixed. Certainly, the approach proposed in reference [9] can be used as an independent check for the results, in the high temperature limit, derived by the Bethe ansatz, but we must first show its applicability; we intend to do so by recovering the analytical expressions of two limiting cases known in the literature.

The aim of this paper is to highlight the alternative method of reference [9] which evaluates the $\beta$-expansion of the grand potential per site to translationally invariant chain models with first neighbors interaction and subject to a periodic boundary condition [9]. In order to exemplify the method, we consider the XXZ model in Ising limit case $(t=0)$ and free fermion case $(\Delta=0$ and $h=0)$. We compare our results $(t=0)$ with [7] and ( $\Delta=0$ and $h=0$ ) with [8] reobtaining their results.

In section 2 , we summarize the results of reference 
[9], and in section 3 the method developed there is applied to the Heisenberg XXZ model in both the Ising limit case and the free fermion case. We recover the known results in literature. In section 4 we draw our main conclusions.

\section{Grand Potential per Site for a Chain Model}

Here we present a survey of an alternative method for calculating the coefficients of the high temperature expansion of the thermodynamic functions of translationally invariant chain models with interaction between nearest neighbors and satisfying a periodic boundary condition [9].

Let us consider a one-dimensional regular lattice (a periodic chain) with $N$ sites, so that its Hilbert space is simply $\mathcal{H}^{(N)} \stackrel{N}{\otimes} \mathcal{H}, \mathcal{H}$ being the irreducible representation at one site, including all its degrees to freedom. The dimension of this Hilbert space is $\operatorname{dim} \mathcal{H}^{(N)}=\operatorname{tr}_{N}(\mathbb{1})$. The notation $\operatorname{tr}_{N}$ means the trace over all $N$ sites and their internal degrees of freedom, e.g. spin.

The grand canonical partition function of a quantum system in the chain with $N$ sites is given by

$$
\mathcal{Z}_{N}(\beta, \mu)=\operatorname{tr}_{N}\left(\mathrm{e}^{-\beta \mathbb{K}}\right),
$$

where $\mathbb{K}=\mathbb{H}-\mu \mathbb{N}, \mu$ being the chemical potential and $\mathbb{N}$ being an operator that commutes with the Hamiltonian of the system. The expansion of $\mathcal{Z}_{N}(\beta, \mu)$ around $\beta=0$ is

$$
\mathcal{Z}_{N}(\beta, \mu)=\operatorname{tr}_{N}(\mathbb{1})+\sum_{n=1}^{\infty}(-\beta)^{n} \frac{\operatorname{tr}_{N}\left(\mathbb{K}^{n}\right)}{n !}
$$

Let $\mathbf{A}$ be any operator that acts on $\mathcal{H}^{(M)}$ where $M \leq N$. We define $\langle\mathbf{A}\rangle \equiv \frac{\operatorname{tr}_{M}(\mathbf{A})}{\operatorname{tr}_{M}(\mathbf{1})}$, for any dimension of $\mathcal{H}^{(M)}$, as the normalized trace of operator $\mathbf{A}$.

Using the definition of normalized trace, eq.(2) becomes

$$
\mathcal{Z}_{N}(\beta, \mu)=\operatorname{tr}_{N}(\mathbb{1})\left\{1+\sum_{n=1}^{\infty}(-\beta)^{n} \frac{\left\langle\mathbb{K}^{n}\right\rangle}{n !}\right\}
$$

We consider a general Hamiltonian $\mathbb{H}$ subject to two constraints: the interaction is only between first neighbors and the Hamiltonian $\mathbb{H}$ is translationally invariant. The most general operator $\mathbb{K}$ satisfying both conditions is

$$
\mathbb{K}=\sum_{i=1}^{N} \widetilde{\mathbf{K}}_{i, i+1}
$$

where

$$
\widetilde{\mathbf{K}}_{i, i+1}=\mathbf{1}_{1} \otimes \ldots \otimes \mathbf{1}_{i-1} \otimes \mathbf{K}_{i, i+1} \otimes \mathbf{1}_{i+2} \otimes \ldots \otimes \mathbf{1}_{N}
$$

and $\mathbf{K}_{i, i+1} \in \mathcal{H}_{i} \otimes \mathcal{H}_{i+1}$. We use the notation $\mathbf{1}_{i} \in \mathcal{H}_{i}$ for the identity matrix in the irreducible sub-space of the $i^{\text {th }}$ particle.

In reference [9] we showed that the coefficients $\left\langle\mathbb{K}^{n}\right\rangle$ in eq.(2) can be written, for arbitrary $n$, as

$$
\frac{\left\langle\mathbb{K}^{n}\right\rangle}{n !}=\sum_{r=1}^{[n, N]} \sum_{m=r}^{[n, N]} \frac{N}{r}\left(\begin{array}{c}
N-m-1 \\
r-1
\end{array}\right) \mathrm{K}_{r, m}^{(n)} .
$$

The notation $[n, N]$ means $\min (n, N)$ and $\mathrm{K}_{r, m}^{(n)}$ is defined by

$$
\mathrm{K}_{r, m}^{(n)} \equiv \sum_{\left\{n_{i}\right\}}^{n} \sum_{\left\{m_{i}\right\}}^{m} \prod_{j=1}^{r} \mathrm{~K}_{1, m_{j}}^{\left(n_{j}\right)}
$$

where $\sum_{\left\{n_{i}\right\}}^{n}$ " means the restriction: $\sum_{\{i=1\}}^{n}$ " and $n_{i} \neq 0$ for $i=1,2, . ., m$. We also use the notation $\left\{n_{i}\right\} \equiv\left\{n_{1}, n_{2}, \ldots, n_{r}\right\}$ and $\left\{m_{i}\right\} \equiv\left\{m_{1}, m_{2}, \ldots, m_{r}\right\}$. The function $\mathrm{K}_{1, m}^{(n)}$ is defined as

$$
\mathrm{K}_{1, m}^{(n)}=\sum_{\left\{n_{i}\right\}}^{n} "\left\langle\prod_{i=1}^{m} \frac{\mathbf{K}_{i, i+1}^{n_{i}}}{n_{i} !}\right\rangle_{\mathrm{g}}
$$

and each term on the r.h.s. of eq.(8) corresponds to the g-trace of an open connected sub-chain.

In the definition of the function $\mathrm{K}_{1, m}^{(n)}$ we have the g-trace which means

$$
\left\langle\frac{\mathbf{K}_{i_{1}, i_{1}+1}^{n_{1}} \mathbf{K}_{i_{2}, i_{2}+1}^{n_{2}} \ldots \mathbf{K}_{i_{m}, i_{m}+1}^{n_{m}}}{n_{1} ! n_{2} ! \ldots n_{m} !}\right\rangle_{\mathrm{g}} \equiv \frac{1}{n !} \sum_{\mathcal{P}}\left\langle\mathcal{P}\left(\mathbf{K}_{i_{1}, i_{1}+1}^{n_{1}}, \mathbf{K}_{i_{2}, i_{2}+1}^{n_{2}}, \ldots, \mathbf{K}_{i_{m}, i_{m}+1}^{n_{m}}\right)\right\rangle
$$


where $\sum_{i=1}^{m} n_{i}=n$ with $n_{i} \neq 0$ and the indices $i_{k}, k=1 . . m$ have distinct values. The notation $\left\langle\mathcal{P}\left(\mathbf{K}_{i_{1}, i_{1}+1}, \mathbf{K}_{i_{2}, i_{2}+1}, \ldots, \mathbf{K}_{i_{m}, i_{m}+1}\right)\right\rangle$ represents all the permutations of the $n$ operators $\left\{\mathbf{K}_{i_{1}, i_{1}+1}, \mathbf{K}_{i_{2}, i_{2}+1}, \ldots, \mathbf{K}_{i_{m}, i_{m}+1}\right\}$.

We show in reference [9], that the grand potential per site $\mathcal{W}(\beta, \mu)$,

$$
\mathcal{W}(\beta, \mu)=-\lim _{N \rightarrow \infty} \frac{1}{N \beta} \ln \mathcal{Z}_{N}(\beta, \mu)
$$

in the thermodynamic limit is written as

$$
\mathcal{W}(\beta, \mu)=-\frac{1}{\beta}\left\{\ln \left(\operatorname{tr}_{1}(\mathbf{1})\right)+\ln (1+\xi)\right\}
$$

where

$$
\xi=\left.\sum_{n=0}^{\infty} \frac{\mathrm{d}^{n}}{\mathrm{~d} \lambda^{n}}\left(\frac{\varphi(\lambda)^{n+1}}{(n+1) !}\right)\right|_{\lambda=1}
$$

where $\lambda$ is a parameter, and the auxiliary function $\varphi(\lambda)$ is equal to

$$
\varphi(\lambda)=\sum_{m=1}^{\infty} \frac{\Gamma_{m}}{\lambda^{m}}
$$

and

$$
\Gamma_{m} \equiv \sum_{n=m}^{\infty}(-\beta)^{n} \mathrm{~K}_{1, m}^{(n)}
$$

From eqs.(11)-(14) we see that only the open connected sub-chains contribute to the grand potential per site, in the thermodynamic limit. The weight of each sub-chain in the $\beta$-expansion of $\mathcal{W}(\beta, \mu)$ is already presented in eq.(11).

\section{The Heisenberg XXZ Model}

Let us consider the Hamiltonian of the well known anisotropic one-dimensional Heisenberg XXZ model with spin- $\frac{1}{2}[1,10,11]$

$$
\mathbb{H}=\sum_{j=1}^{N}\left(S_{j}^{x} S_{j+1}^{x}+S_{j}^{y} S_{j+1}^{y}+\Delta S_{j}^{z} S_{j+1}^{z}-2 h S_{j}^{z}\right),
$$

where $h$ is the external magnetic field and $\left(S_{j}^{x}, S_{j}^{y}, S_{j}^{z}\right)$ are spin- $\frac{1}{2}$ operators the $j^{\text {th }}$ site in a periodic chain with $N$ space sites; $\Delta$ is called the anisotropy parameter and for $\Delta>0(\Delta<0)$ we have a repulsive (attractive) interaction core. The case $\Delta=1(\Delta=-1)$ corresponds to the isotropic antiferromagnetic (ferromagnetic) Heisenberg model with ground state fully polarized. Through the Jordan-Wigner transformation[12], the Hamiltonian (15) is mapped onto the spinless fermionic model, whose Hamiltonian is

$$
\mathbb{H}=\sum_{j=1}^{N}\left(t\left(\mathbf{a}_{j}^{\dagger} \mathbf{a}_{j+1}+\mathbf{a}_{j+1}^{\dagger} \mathbf{a}_{j}\right)+V \mathbf{n}_{j} \mathbf{n}_{j+1}+E \mathbf{n}_{j}\right)+N\left(h+\frac{\Delta}{2}\right),
$$

where $V=2 \Delta, E=-2 h-2 \Delta, \mathbf{n}_{i}=\mathbf{a}_{i}^{\dagger} \mathbf{a}_{i}$ and $\mathbf{a}_{i}^{\dagger}\left(\mathbf{a}_{i}\right)$ is the fermionic creation (annihilation) operator at the $j^{\text {th }}$ site. The operators $\mathbf{a}_{i}^{\dagger}$ and $\mathbf{a}_{i}$ satisfy the usual anticommutation relations of the fermionic fields. In writng hamiltonian (16) we consider the situation where the number of down-spins is odd [1]. However we point out that this choice does not restrict our results since we are interested in the thermodynamic limit of the model. The hopping constant $t$ is included in the hamiltonian (16) only to help counting the powers of terms that contribute to a given order $\beta^{n}$ in the $\beta$-expansion of the free energy. Throughout the calculations, we have taken $t=1$. The term $N\left(h+\frac{\Delta}{2}\right)$ leads to a shift in the energy of the ground state of the model.

The Hamiltonian (16) has two liming cases: the Ising model $(t=0)$ and the free fermion case $(\Delta=0$ and $h=0$ ), that have closed analytical expressions for their respective free energies. Now we are going to apply the method outlined in section 2 to each of these models.

\section{III.1 The Ising Limit Case $(t=0)$}

For the sake of simplicity, we will drop along the calculations the contribution of the constant term on the r.h.s. of the Hamiltonian (16); it will be recovered in the final expression of the free energy. The Hamiltonian of the Ising model is:

$$
\mathbb{V}=\sum_{j=1}^{N}\left(V \mathbf{n}_{j} \mathbf{n}_{j+1}+E \mathbf{n}_{j}\right)
$$

To obtain the auxiliary function $\varphi(\lambda)$ from which the free energy in the thermodynamic limit will be derived, 
we must calculate $\mathrm{V}_{1, m}^{(n)}$. Applying eq.(8) to this model, we have

$$
\mathrm{V}_{1, m}^{(n)}=\sum_{\left\{n_{i}\right\}}^{n}\left\langle\prod_{i=1}^{m} \frac{\left(V \mathbf{n}_{i} \mathbf{n}_{i+1}+E \mathbf{n}_{i}\right)^{n_{i}}}{n_{i} !}\right\rangle
$$

To this particular model the g-traces are already the normalized traces. In order to evaluate the normalized traces we apply the property of the operator number of a fermionic particle: $\left(\mathbf{n}_{i}\right)^{m}=\mathbf{n}_{i}, m \neq 0$. In doing that we get

$$
\mathrm{V}_{1, m}^{(n)}=\sum_{\left\{n_{i}\right\}}^{n}\left\langle\mathbf{n}_{1}\left(\prod_{i=1}^{m-1} \frac{\left(V \mathbf{n}_{i+1}+E\right)^{n_{i}} \mathbf{n}_{i+1}}{n_{i} !}\right) \frac{\left(V \mathbf{n}_{m+1}+E\right)^{n_{m}}}{n_{m} !}\right\rangle .
$$

We should remember that the normalized trace of the number operator in this model is $\left\langle\mathbf{n}_{i}\right\rangle=\frac{1}{2}$. Due to the fact that all operators in Hamiltonian(17) commute among themselves, we expand the terms in the product of eq.(19) using the binomial expansion, obtaining

$$
\mathrm{V}_{1, m}^{(n)}=\frac{1}{2^{m}} \sum_{\left\{n_{i}\right\}}^{n}\left(\prod_{i=1}^{m-1} \frac{(-2 h)^{n_{i}}}{n_{i} !}\right) \frac{1}{2}\left\{\frac{(-2 h)^{n_{m}}}{n_{m} !}+\frac{(2(-h-\Delta))^{n_{m}}}{n_{m} !}\right\} .
$$

In order to calculate the $\Gamma_{m}$ functions (see eq.(14)) we perform the summation over $n=1,2, \ldots, \infty$. The restricted sums over the indices $\left\{m_{i}\right\}$ are easily done if we recognized that in the thermodynamic limit they can be substituted by $m$ independent sums with each index $\left\{m_{i}\right\}$ varying from 1 to $\infty$. Then the $\Gamma_{m}$ functions can be written as a product of sums and

$$
\Gamma_{m}=\left(\frac{\mathrm{e}^{2 \beta h}-1}{2}\right)^{m-1} \frac{\left(\mathrm{e}^{2 \beta h}+\mathrm{e}^{2 \beta(h+\Delta)}-2\right)}{4} .
$$

Our next step is to obtain the auxiliary function $\varphi(\lambda)$, substituting eq.(21) in eq.(13), that is

$$
\varphi(\lambda)=\frac{\left(\mathrm{e}^{2 \beta h}+\mathrm{e}^{2 \beta(h+\Delta)}-2\right)}{4 \lambda} \sum_{m=1}^{\infty}\left(\frac{\mathrm{e}^{2 \beta h}-1}{2 \lambda}\right)^{m-1} .
$$

The summation on the r.h.s. of eq.(22) is easily done and we obtain the following expression

$$
\varphi(\lambda)=\frac{\left(\mathrm{e}^{2 \beta h}+\mathrm{e}^{2 \beta(h+\Delta)}-2\right)}{4}\left(\frac{1}{\lambda-\frac{\mathrm{e}^{\beta h}-1}{2}}\right) .
$$

Finally, substituting this $\varphi(\lambda)$ into the definition of $\xi$ (see eq.(12)), we obtain

$$
\xi=\frac{1}{2}\left\{\frac{\mathrm{e}^{2 \beta h}-3}{2}+\sqrt{\left(\frac{\mathrm{e}^{2 \beta h}-1}{2}\right)^{2}+\mathrm{e}^{2 \beta(h+\Delta)}}\right\} .
$$

At this stage of our calculations, we put back the contribution from the shift of the energy of the ground state $\left(\mathrm{e}^{-\beta\left(h+\frac{\Delta}{2}\right)}\right)$ in the expression of the free energy of the Ising model

$$
\begin{aligned}
\mathcal{W}_{I}(\beta) & =-\frac{1}{\beta}(\ln (2)+\ln (1+\xi)) \\
& =-\frac{1}{\beta} \ln \left(\mathrm{e}^{\frac{-\beta \Delta}{2}} \cosh (\beta h)+\sqrt{\mathrm{e}^{-\beta \Delta} \sinh ^{2}(\beta h)+\mathrm{e}^{\beta \Delta}}\right) .
\end{aligned}
$$


Expression (25) coincides with the results found in the literature[7].

\section{III.2 Free Fermion Model $(\Delta=0$ and $h=0$ )}

The free fermion model in one-dimension comes from the Heisenberg Hamiltonian(16) with $\Delta=0$ and $h=0$.

For this model, the $\mathrm{H}_{1, m}^{(n)}$ are null when $n$ is odd. For this reason, our expansion is done only for even values of $n$. Below we present the calculated expressions for $\mathrm{H}_{1, m}^{(n)}$ up to $n=10$ :

$$
\begin{aligned}
\mathrm{H}_{1,1}^{(2)} & =\frac{t^{2}}{4} \\
\mathrm{H}_{1,1}^{(4)} & =\frac{t^{4}}{48} \quad \mathrm{H}_{1,2}^{(4)}=\frac{t^{4}}{24} \\
\mathrm{H}_{1,1}^{(6)} & =\frac{t^{6}}{1440} \quad \mathrm{H}_{1,2}^{(6)}=\frac{t^{6}}{240} \quad \mathrm{H}_{1,3}^{(6)}=\frac{7 t^{6}}{960} \\
\mathrm{H}_{1,1}^{(8)} & =\frac{t^{8}}{80640} \quad \mathrm{H}_{1,2}^{(8)}=\frac{t^{8}}{5760} \quad \mathrm{H}_{1,3}^{(8)}=\frac{43 t^{8}}{40320} \quad \mathrm{H}_{1,4}^{(8)}=\frac{17 t^{8}}{13440} \\
\mathrm{H}_{1,1}^{(10)} & =\frac{t^{10}}{7257600} \quad \mathrm{H}_{1,2}^{(10)}=\frac{t^{10}}{241920} \quad \mathrm{H}_{1,3}^{(10)}=\frac{t^{10}}{13824} \quad \mathrm{H}_{1,4}^{(10)}=\frac{113 t^{10}}{483840} \quad \mathrm{H}_{1,5}^{(10)}=\frac{319 t^{10}}{1451520},
\end{aligned}
$$

which give us

$$
\begin{aligned}
& \Gamma_{1}=\frac{(\beta t)^{2}}{4}+\frac{(\beta t)^{4}}{48}+\frac{(\beta t)^{6}}{1440}+\frac{(\beta t)^{8}}{80640}+\frac{(\beta t)^{10}}{7257600}+\mathcal{O}\left(\beta^{11}\right) \\
& \Gamma_{2}=\frac{(\beta t)^{4}}{24}+\frac{(\beta t)^{6}}{240}+\frac{(\beta t)^{8}}{5760}+\frac{(\beta t)^{10}}{241920}+\mathcal{O}\left(\beta^{11}\right) \\
& \Gamma_{3}=\frac{7(\beta t)^{6}}{960}+\frac{43(\beta t)^{8}}{40320}+\frac{(\beta t)^{10}}{13824}+\mathcal{O}\left(\beta^{11}\right), \\
& \Gamma_{4}=\frac{17(\beta t)^{8}}{13440}+\frac{113(\beta t)^{10}}{483840}+\mathcal{O}\left(\beta^{11}\right) \\
& \Gamma_{5}=\frac{319(\beta t)^{10}}{1451520}+\mathcal{O}\left(\beta^{11}\right) .
\end{aligned}
$$

From the results $(27)$ we obtain the function $\varphi(\lambda)$ for the model. The sum of sub-chains (see eq.(12)) that contribute to $\mathcal{W}_{F}(\beta, \mu)$ is

$$
\xi=\frac{1}{4}(\beta t)^{2}+\frac{1}{576}(\beta t)^{6}-\frac{1}{2304}(\beta t)^{8}+\frac{29}{230400}(\beta t)^{10}+\mathcal{O}\left(\beta^{11}\right) .
$$

From eq.(11), we get

$$
\mathcal{W}_{F}(\beta)=-\frac{\ln (2)}{\beta}-\frac{1}{2 \beta}\left\{\frac{1}{2} \frac{(\beta t)^{2}}{1 !^{2}}-\frac{1}{4} \frac{(\beta t)^{4}}{2 !^{2}}+\frac{1}{2} \frac{(\beta t)^{6}}{3 !^{2}}-\frac{17}{8} \frac{(\beta t)^{8}}{4 !^{2}}+\frac{31}{2} \frac{(\beta t)^{10}}{5 !^{2}}+\mathcal{O}\left(\beta^{11}\right)\right\} .
$$

We recognize that the coefficients in expansion (29) can be written in terms of the Euler's number $\mathrm{E}_{n}(x)$ evaluated at $x=1$. Extending our results, using Euler's number for arbitrary $n$, we obtain

$$
\mathcal{W}_{F}(\beta)=-\frac{\ln (2)}{\beta}-\frac{1}{2 \beta} \sum_{n=1}^{\infty} \mathrm{E}_{2 n-1}(1) \frac{(\beta t)^{2 n}}{n !^{2}}=-\frac{1}{2 \pi \beta} \int_{0}^{2 \pi} \mathrm{d} \theta \ln \left(1+\mathrm{e}^{2 \beta t \cos (\theta)}\right) .
$$

The integral on the r.h.s. of eq.(30) is just the well known result for the free fermion model[8]. 


\section{Conclusions}

Some chain models have the property of integrability, and their exact thermodynamics is usually obtained via Bethe ansatz. The thermodynamic functions are written in terms of NLIE. The analysis of their thermodynamic properties implies a very involved numerical manipulation of those NLIE. It is important that we have alternative formalisms, preferably algebraic and independent. We have thereby the possibility of testing methods and known results. One of these methods was showed in [9].

As an illustrative application of such method, we consider the Heisenberg XXZ spin- $\frac{1}{2}$ chain model, and we derive its thermodynamic potential for two limit cases: the fermionic spinless version of the onedimensional Ising model (when $t=0$ ) and the free fermion case $(\Delta=0)$. For the Ising model, we recover the result of reference [7] by re-summing the $\beta$ expansion series of the thermodynamic potential. For the free fermion model, we calculate $\mathcal{W}_{F}(\beta, \mu)$ up to order $(\beta t)^{10}$ and show that all terms agree with known results[8]. The important point is that the results are analytical and this technique can be applied to any translationally invariant chain models (fermionic or bosonic) with first neighbors interaction, subject to periodic boundary condition.

Finally we want to mention that it is in progress the application of the present approach to derive the high temperature expansion of the spin-1 Heisenberg model. We point out that the thermodynamics of this model can not be studied by the Bethe ansatz method once it does not owe the integrability property.

\section{Acknowledgments}

O. R. thanks CAPES for financial support. M.T.T and S.M. de S. thank $\mathrm{CNPq}$ for partial financial support. S.M. de S. thanks FAPEMIG for partial financial support. M.T.T. also thanks FAPERJ for partial financial support.

\section{References}

[1] M. Takahashi, Thermodynamics of One-Dimensional Solvable Models, Cambridge Univ. Press. (1999).

[2] J. Hubbard, Proc. Roy. Soc. A277 (1963) 237; A281 (1964) 401;

M. Gutzwiller, Phys. Rev. Lett. 10, 159 (1963); Phys. Rev. A137, 1726 (1965).

[3] A. Klümper and R.Z. Bariev, Nucl. Phys. B 458, [FS] 623 (1996).

[4] M.J. Martins and P.B. Ramos, Nucl. Phys. B 522, [FS] 413 (1998).

[5] G. Jüttner, A. Klümper and J. Suzuki, Nucl. Phys. B522, [FS] 471 (1998)

[6] C. Destri and H. J. de Vega, Nucl. Phys. B 438 [FS], 413 (1995).

[7] R. J. Baxter, Exactly Solved Models in Statistical Mechanics, Academic Press, 1982.

[8] A. Klümper, Z. Phys. B91, 507 (1993).

[9] Onofre Rojas, S. M. de Souza and M. T. Thomaz, Chain Model at High Temperature Expansion, a revised version of hep-ph/0012368, 2001, accepted to publication in Journal of Mathematical Physics.

[10] M. Gaudin, Phys. Rev. Lett. 26, 1301 (1971).

[11] A. Luther, Phys. Rev. B 14, 2153 (1976).

[12] The Jordan-Wigner transformation is given by: $S_{m}^{-}=\mathrm{e}^{i \pi \sum_{j=1}^{m-1} \mathbf{a}_{j}^{\dagger} \mathbf{a}_{j}} \mathbf{a}_{m}$ and $S_{m}^{+}=\left(S_{m}^{-}\right)^{\dagger}$ with $S_{m}^{ \pm}=$ $S_{m}^{x} \pm i S_{m}^{y}$ and $S_{m}^{z}=2 \mathbf{a}_{m}^{\dagger} \mathbf{a}_{m}-1=2 \mathbf{n}_{m}-1$. 